\title{
Analysis of patient characteristics, health care costs by surgical venue, and opioid utilization for common orthopedic procedures in the United States
}

Fanta Waterman, PhD, MPH; Miriam Cisternas, MA; Stephanie Korrer, MPH; and Annikka Wilson, MPH

\section{What is already known about this subject}

- Transitioning surgical procedures from inpatient venues to hospital outpatient departments (HOPDs) and ambulatory surgical centers (ASCs) can result in considerable cost savings.

- Lower costs with orthopedic procedures performed in outpatient surgery sites have been previously reported.

\section{ABSTRACT \\ BACKGROUND: Orthopedic surgery can be performed in hospital outpatient depart- ments (HOPDs) and ambulatory surgical centers (ASCs), as well as in traditional inpatient venues. Patients who undergo orthopedic surgery may be prescribed opi- oids for the management of postsurgical pain. However, the association between surgery venue, postsurgical opioid use, and health care costs remains unclear. \\ OBJECTIVE: To compare postsurgical opioid use and health care costs associated with 6 different orthopedic surgical procedures performed at inpatient, ASC, and HOPD venues.}

\author{
What this study adds \\ - Compared with procedures at inpatient \\ venues, procedures at HOPDs and \\ ASCs were associated with statistically \\ significant lower all-cause costs on the \\ day of surgery through 30 and 90 days \\ afterward for 6 orthopedic procedures \\ even after adjusting for cohort, surgery \\ year, demographic characteristics, \\ baseline Quan-Charlson Comorbidity \\ Index, and any opioid use within 90 days \\ before the procedure ( 30 days: $14 \%$ and \\ $27 \%$ lower, respectively; 90 days: $13 \%$ \\ and $26 \%$ lower, respectively). \\ - Adjusted percentages of persistent \\ postsurgical opioid use were lowest in \\ ASCs (18\%) compared with HOPDs (24\%) \\ or inpatient venues (26\%).
}

METHODS: Using the Optum Research Database, this retrospective study analyzed commercial health care claims from adult patients in the United States undergoing specific orthopedic procedures (total knee arthroplasty, partial knee arthroplasty, total hip arthroplasty, total shoulder arthroplasty, rotator cuff repair, and lumbar spinal fusion) between April 1, 2012, and December 31. 2017. The date of the first procedure in that period was the index date; continuous insurance coverage for 12 months before the index date (baseline period) to 6 months following the index date (postsurgical period, which includes the index date) was required. Opioid use and all-cause costs were measured in the postsurgical period. Baseline patient characteristics included

\section{Author affiliations \\ Fanta Waterman, PhD, MPH, Pacira \\ BioSciences, Parsippany, NJ, and Serrette Brown Research and Consulting, Danbury, CT. Miriam Cisternas, MA; Stephanie Korrer, $\mathrm{MPH}$; and Annikka Wilson, MPH, Health Economics and Outcomes Research, Optum, Eden Prairie, MN.}

\section{AUTHOR CORRESPONDENCE:}

Fanta Waterman, 203.493.6687;

fanta.waterman@gmail.com

J Manag Care Spec Pharm. 2021;27(5):586-95

Copyright $\odot 2021$, Academy of Managed Care Pharmacy. All rights reserved.

demographics, Quan-Charlson Comorbidity Index, and opioid use. Multivariable analysis identified factors influencing postsurgical costs and persistent opioid use (defined as $\geq 1$ opioid fill within 3 days after surgery [or discharge for inpatient stay] and $\geq 1$ additional opioid fill during the postsurgical period at least 90 days after the index date).

RESULTS: The sample included 126,172 patients (mean age, 58 years; $49 \%$ female). Overall, most procedures were performed at inpatient venues (68\%), followed by HOPDs (18\%) and ASCs (14\%); the percentage of procedures performed at ASCs increased from $12 \%$ to $17 \%$ from 2012 to 2017 . Patients whose procedures were performed at ASCs reported the lowest adjusted percentage of 
persistent opioid use following the procedure (18\%) compared with those with procedures performed at HOPDs $(24 \%)$ or inpatient venues (26\%). Adjusted 30 -day costs were $14 \%$ and $27 \%$ lower for patients with procedures in HOPDs and ASCs, respectively, compared with inpatient venues $(P<0.001$ for both), and adjusted costs over the first 90 days were similar.

CONCLUSIONS: All-cause costs on the day of surgery through 30 days after surgery for these 6 orthopedic procedures were significantly lower in HOPDs and ASCs compared with inpatient venues, even after adjustment for cohort, surgery year, demographic characteristics, baseline Quan-Charlson Comorbidity Index, and any opioid use within 90 days before the procedure. Additionally, patients undergoing orthopedic surgery at ASCs had the lowest adjusted percentage of persistent opioid use compared with those undergoing surgery at HOPDs or inpatient venues. Migration of certain orthopedic procedures from inpatient venues to HOPDs or ASCs may reduce health care costs and decrease the potential for persistent opioid use.

Outpatient surgical sites, such as ambulatory surgical centers (ASCs) and hospital outpatient departments (HOPDs), have emerged as alternatives to traditional inpatient hospital sites for performing invasive surgical procedures, ${ }^{1}$ with approximately 13.5 million procedures performed at outpatient sites in 2017. ${ }^{2}$ Advances in surgical techniques and regional anesthesia have facilitated the transition of surgical procedures from the inpatient to the outpatient setting, including orthopedic procedures such as hip and knee replacements. ${ }^{1,3,4}$ Indeed, projections estimate that more than half of hip and knee replacements in the United States will be performed at outpatient sites by 2028, and a larger proportion of eligible patients will be shifted to these sites each year. $^{5}$

One of the primary benefits of migrating orthopedic procedures from inpatient venues to ASCs and HOPDs is the potential for considerable cost savings. A retrospective review of medical records found that undergoing total hip arthroplasty (THA) in the outpatient setting was associated with an average of $\$ 6,798$ lower costs (billed charges) compared with undergoing procedures by the same surgeon in an inpatient setting of the same hospital. ${ }^{6}$ The lower costs of surgery observed in the outpatient setting may be explained, in part, by elimination of certain costs acquired with inpatient stays, including the cost of hospital rooms, physical therapy, and occupational therapy. ${ }^{7}$ Furthermore, laboratory charges, cost of medication, and cost of surgical supplies are notably lower in outpatient surgery centers than in inpatient venues. ${ }^{7}$ The economic benefits of procedures performed at outpatient venues may also extend beyond the surgical procedure itself. For example, an analysis of commercial insurance claims for patients undergoing total knee arthroplasty (TKA) or THA in which ASC and inpatient procedures were propensity score matched on a clinical risk profile found that, in addition to lower costs (payments) for the ASC procedures compared with inpatient procedures, postsurgical costs (all postsurgical care payments through 90 days after surgery) at ASCs were 8\% and 21\% lower than postsurgical cost outcomes at an inpatient setting for TKA and THA procedures, respectively. ${ }^{8}$

Although economic benefits favor outpatient surgery centers for orthopedic procedures, these benefits may also be reflective of the condition of patients eligible for outpatient surgery, who are often younger and generally present with minimal notable medical history and who presumably are at low risk of experiencing surgical complications. ${ }^{9,10}$ It is unknown whether elements of postsurgical care, such as prescription opioid use for postsurgical pain control, differ between inpatient and outpatient surgical venues. Opioids are commonly prescribed to manage pain after surgery. ${ }^{11}$ However, given the high levels of pain after orthopedic procedures, minimizing opioid exposure and potential misuse is imperative for overall patient wellbeing. ${ }^{12}$ Therefore, it would be valuable to ascertain whether surgical venues influence the likelihood of developing persistent postsurgical opioid use. This retrospective review of commercial insurance claims analyzed postsurgical opioid use in patients undergoing 1 of 6 orthopedic procedures and compared combined surgical and postsurgical health care costs as well as persistent postsurgical opioid use across inpatient venues, HOPDs, and ASCs, both descriptively and adjusted for differences in patient characteristics.

\section{Methods}

\section{DATA SOURCE}

Data were extracted from the Optum Research Database, a proprietary health care claims research database. The data were fully deidentified before access by the research team and were used in compliance with Health Insurance Portability and Accountability Act regulations. All patientlevel identifiers in the Optum Research Database are encrypted to protect patient privacy. In accordance with the Health Insurance Portability and Accountability Act, institutional review board approval was not required. The database contains medical and pharmacy claims data with linked enrollment information. Medical claims include services from all venues, including inpatient, outpatient, emergency room, surgical center, and physician offices. Medical claims contain diagnosis codes as defined in the 
International Classification of Diseases, Ninth/Tenth Revision, Clinical Modification (ICD-9/10-CM). Procedures are coded per the ICD-9/10-CM, current procedural terminology, and Healthcare Common Procedural Coding System. Outpatient pharmacy claims include the national drug code, fill date, quantity of drug dispensed, and days of supply. Both medical and pharmacy claims are amounts paid by both health care insurers and patients.

\section{STUDY DESIGN AND PATIENT SELECTION}

This retrospective claims analysis identified commercially insured patients undergoing 6 orthopedic procedures (TKA, partial knee arthroplasty [PKA], THA, total shoulder arthroplasty [TSA], rotator cuff repair [RCR], or lumbar spinal fusion [LSF]) between April 1, 2012, and December 31, 2017 (identification period). Current procedural terminology codes are shown in Supplementary Table 1 (available in online article). The date of the first of these procedures was the index date.

Patients were excluded if there was $\geq 1$ procedure performed on the index date. To be included in the study, patients were also required to be aged $\geq 18$ years, have no missing demographic information as of the index date, and have no evidence of the index surgery in the 12-month pre-index period. Additional criteria were continuous insurance coverage with medical and pharmacy benefits from 12 months before (baseline period) to 6 months after (and including) the index date (postsurgical period), no evidence that the surgery was a revision to a procedure based on current procedural terminology modifiers, and surgery restricted to the following venues: hospital acute inpatient, HOPDs, and ASCs. The entire study period was from April 1, 2011, through June 30, 2018, accounting for the 12-month baseline and 6-month postsurgical periods. Mutually exclusive cohorts were created on the basis of the procedure.

\section{BASELINE CHARACTERISTICS}

Demographic characteristics (age, sex, region) and year of surgery were obtained from the claim for the procedure. The Quan-Charlson Comorbidity Index (calculated using diagnosis codes from medical claims) was measured in the baseline period. ${ }^{13}$ Any baseline opioid use was defined as $\geq 1$ filled opioid prescription during the baseline period; patients with and without any opioid use during the baseline period were also referred to as opioid experienced and opioid naive, respectively. Long-term baseline opioid use was defined as having $\geq 90$-day supply of opioids available during the 120-day period before the index date. We identified opioid medications indicated for pain and did not include opioids indicated for medication-assisted treatment.

\section{STUDY OUTCOMES}

The number of patients with any opioid use (defined as $\geq 1$ filled opioid prescription during the postsurgical period) or persistent opioid use (defined as $\geq 1$ opioid fill within 3 days after the surgery [or discharge for inpatient stay] and $\geq 1$ additional opioid fill during the postsurgical period at least 90 days after the index date) was assessed. All-cause health care costs comprised combined health plan and patient-paid amounts for pharmacy and medical (ambulatory, emergency room, inpatient, and other medical costs combined) services. These costs were calculated for the first 30 and 90 days of the postsurgical period and for the entire postsurgical period, adjusting for inflation to 2017 costs using the Consumer Price Index medical care component (claims in 2018 were not adjusted). ${ }^{14}$

\section{STATISTICAL ANALYSIS}

Patient characteristics and outcomes are shown for the total sample and stratified across the 3 venues and across procedure cohorts. Testing for significant differences was completed using chi-square tests for categorical variables and 1-way analysis of variance for continuous variables. Multivariable generalized linear regression models with a gamma distribution and log link assessed the association of costs for the first 30 and 90 days of the postsurgical period with surgery venue, adjusted for cohort, surgery year, demographic characteristics, baseline Quan-Charlson Comorbidity Index, and any opioid use within 90 days before the procedure. Multivariable logistic regression was used to examine the association of persistent postsurgical opioid use with surgery venue and baseline long-term opioid use, adjusted by procedure cohort, surgery year, demographic characteristics, baseline Quan-Charlson Comorbidity Index, and baseline long-term opioid use.

\section{Results}

\section{DEMOGRAPHICS AND BASELINE CHARACTERISTICS}

A total of 217,753 patients were identified, with 126,172 eligible patients included in the final analysis. The mean age was 58 years, with $49 \%$ of the sample being female. Most patients underwent surgery in inpatient venues $(85,626,68 \%)$, followed by HOPDs $(22,928,18 \%)$ and ASCs $(17,618,14 \%$; Figure 1$)$. The most frequently performed procedure in inpatient venues was TKA, and RCR was the most frequently performed procedure in both HOPDs and ASCs (Supplementary Figure 1, available in online article). From 2012 through 2017, the proportion of patients undergoing procedures at ASCs showed a slight increasing trend with a $5 \%$ increase in percentage points over the study period, while the inpatient 


\section{FIGURE 1 Inclusion Criteria for Optum Research Database Claims Across 6 Orthopedic Procedures and 3 Venues}

\section{Inclusion Criteria}

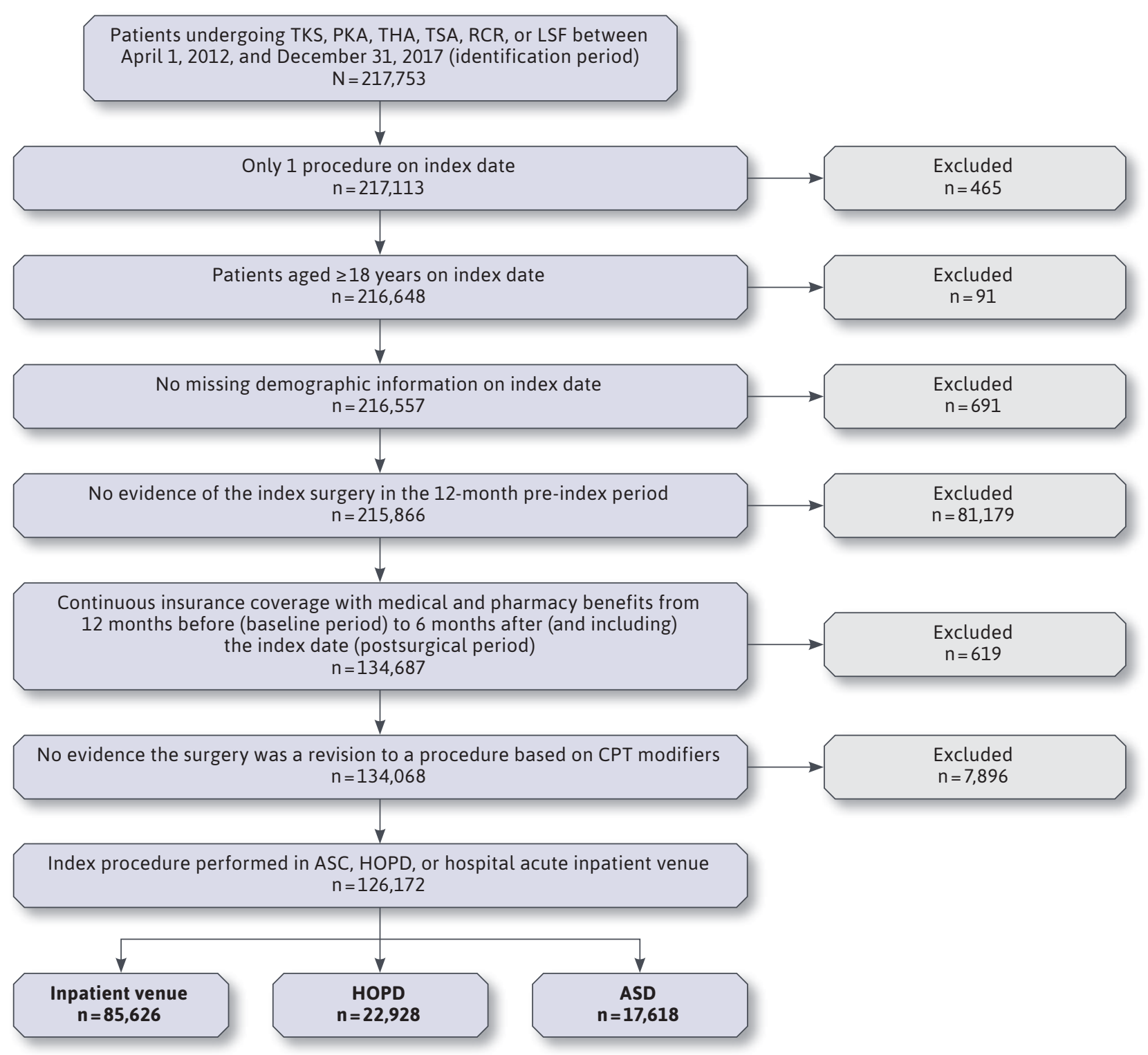

$A S C=$ ambulatory surgical center; $C P T=C$ urrent Procedural Terminology; $H O P D=$ hospital outpatient department; $L S F=$ lumbar spinal fusion; $P K A=$ partial knee arthroplasty; RCR=rotator cuff repair; THA= total hip arthroplasty; TKA= total knee arthroplasty; TSA=total shoulder arthroplasty.

proportion demonstrated a slight decreasing trend with a $6 \%$ decrease in percentage points over the study period; the HOPD proportion remained constant (Supplementary Figure 2, available in online article).
The most common procedure was TKA (40,574, 32\%), followed by RCR (34,207, 27\%), THA (25,585, 20\%), LSF $(18,318$, $15 \%)$, PKA $(4,355,3 \%)$, and TSA $(3,133,2 \%$; Table 1). For all procedures, significant differences across venues were 


\section{TABLE 1 Patient Characteristics by Procedure and Surgical Venue}

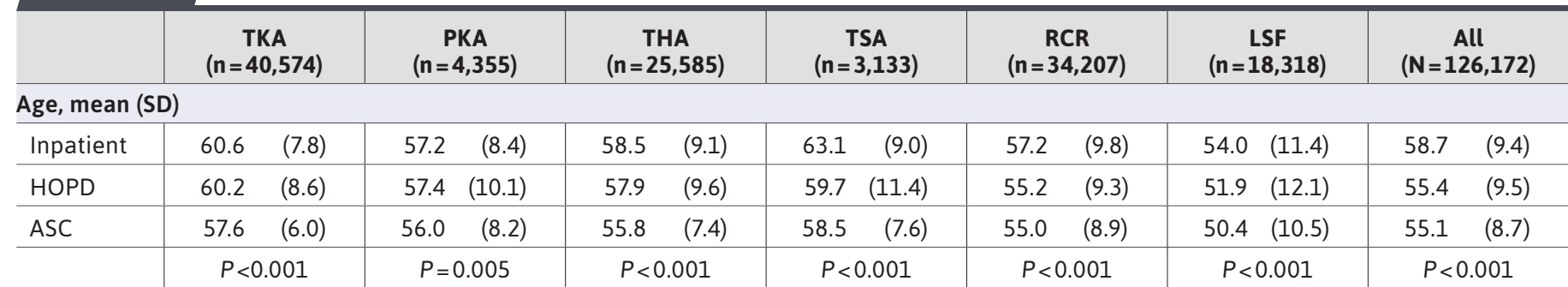

Female, \%

Inpatient

HOPD

\begin{tabular}{l|r} 
HOPD & 56.8 \\
\hline ASC & 51.8 \\
\hline & 52.7 \\
\hline
\end{tabular}

Quan-Charlson Comorbidity Index, mean (SD)

\begin{tabular}{|c|c|c|c|c|c|c|c|}
\hline Inpatient & $0.61 \quad(1.10)$ & $0.50 \quad(0.98)$ & $0.56 \quad(1.13)$ & $0.85 \quad(1.36)$ & $1.04 \quad(1.46)$ & $0.62 \quad(1.12)$ & $0.60 \quad(1.12)$ \\
\hline HOPD & $0.65 \quad(1.17)$ & $0.49 \quad(0.99)$ & $0.50 \quad(1.03)$ & $0.62 \quad(1.14)$ & $0.45 \quad(0.96)$ & $0.55 \quad(1.00)$ & $0.47 \quad(0.97)$ \\
\hline \multirow[t]{2}{*}{ ASC } & $0.39 \quad(0.86)$ & $0.36 \quad(0.77)$ & $0.27 \quad(0.75)$ & $0.31 \quad(0.78)$ & $0.37 \quad(0.84)$ & $0.41 \quad(0.84)$ & $0.37 \quad(0.83)$ \\
\hline & $P<0.001$ & $P=0.004$ & $P<0.001$ & $P<0.001$ & $P<0.001$ & $P<0.001$ & $P<0.001$ \\
\hline
\end{tabular}

Any opioid use at baseline, $\%$

\begin{tabular}{l|c|c|c|c|c|c|c}
\hline Inpatient & 59 & 62 & 61 & 67 & 78 & 82 & 65 \\
\hline HOPD & 62 & 65 & 65 & 63 & 61 & 79 \\
\hline ASC & 62 & 66 & 57 & 64 & 62 & 86 \\
\hline & $P=0.095$ & $P=0.030$ & $P=0.014$ & $P=0.457$ & $P<0.001$ & $P=0.006$ & $P<0.001$ \\
\hline
\end{tabular}

Long-term opioid use at baseline, ${ }^{\text {a }}$

\begin{tabular}{l|c|c|c|c|c|c|c}
\hline Inpatient & 7 & 6 & 8 & 11 & 11 & 21 & 10 \\
\hline HOPD & 7 & 6 & 6 & 9 & 5 & 18 \\
\hline ASC & 4 & 4 & 2 & 6 & 4 & 18 \\
\hline & $P=0.005$ & $P=0.097$ & $P<0.001$ & $P=0.262$ & $P<0.001$ & $P=0.037$ & $P<0.001$ \\
\hline
\end{tabular}

Note: All P values are the result of analysis of variance across surgical venues and do not indicate statistical significance between any 2 venues.

${ }^{a}$ Defined as having $\geq 90$-day supply of opioids during the 120-day period before the index date.

$A S C=$ ambulatory surgical center; $H O P D=$ hospital outpatient department; $L S F=$ lumbar spinal fusion; $P K A=$ partial knee arthroplasty; $R C R=$ rotator cuff repair; $S D=$ standard deviation; THA = total hip arthroplasty; TKA=total knee arthroplasty; TSA = total shoulder arthroplasty.

observed for age, sex, and Quan-Charlson Comorbidity Index, with the exception of no significant difference for sex across surgical venues for PKA and TSA. Numerically higher values for mean age, percentage of female patients, and mean Quan-Charlson Comorbidity Index were observed among patients undergoing surgery at inpatient venues. While baseline opioid use ranged from $63 \%$ to $65 \%$ across the 3 surgical venues, long-term baseline use ranged from $4 \%$ to $10 \%$. The highest percentage of patients with any baseline opioid exposure or long-term opioid exposure was at inpatient venues. A list of opioids included in the study is shown in Supplementary Table 2 (available in online article).

\section{POSTSURGICAL OPIOID USE}

The overall prevalence of any postsurgical opioid use was high in both opioid-naive and opioid-experienced patients (91\% and 93\%, respectively). Unadjusted percentages of persistent postsurgical opioid use were lowest in ASCs (ASCs: 16\%; inpatient: 27\%; HOPDs: 23\%) and were approximately 3 times higher among patients with long-term baseline opioid use than those without it (68\% vs. 21\%; Figure 2). Adjusting for covariates did not substantially alter estimates of persistent opioid use either by venue or long-term baseline opioid use. Unadjusted prevalence of persistent opioid use was numerically higher in patients with baseline 


\section{FIGURE 2 Unadjusted and Adjusted Persistent Opioid Use in the Postsurgical Period by Venue and Baseline Long-Term Opioid Use}

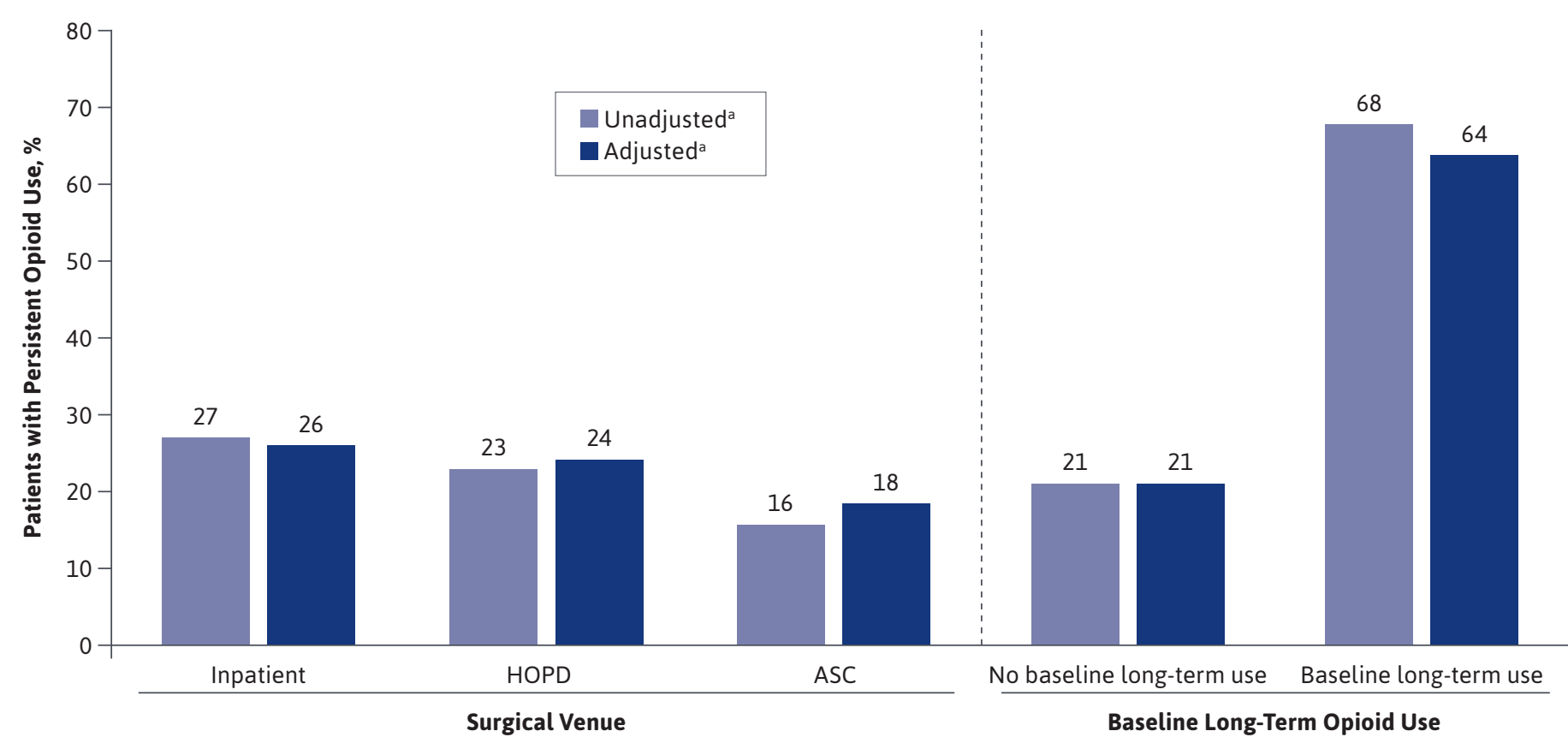

Note: This model was also adjusted for cohort, surgery year, demographic characteristics, and Quan-Charlson Comorbidity Index.

apairwise differences among both unadjusted and adjusted percentages were statistically significant $(P<0.001)$.

$A S C=$ ambulatory surgical center; HOPD = hospital outpatient department.

long-term use versus those without baseline long-term use for inpatient (34\% vs. 14\%), HOPD (30\% vs. 11\%), and ASC (20\% vs. 10\%) venues (Supplementary Figure 3, available in online article). Parameter estimates for all covariates are shown in Supplementary Table 3 (available in online article).

\section{HEALTH CARE COSTS}

Significant differences in all-cause health care costs were identified among surgical venues. Procedures incurring the highest total medical and pharmacy costs during the first 30 days of the postsurgical period were performed in inpatient settings, followed by HOPDs and ASCs. When examining surgical types separately, LSF performed in the inpatient setting was associated with the highest total medical and pharmacy cost $(\$ 81,963)$, while RCR in the ASC setting was associated with the lowest total medical and pharmacy cost $(\$ 14,836$; Table 2). Similar patterns were observed for total medical and pharmacy health care costs during the entire 6-month postsurgical period, with LSF in the inpatient setting associated with highest total medical and pharmacy health care costs $(\$ 92,082)$ and RCR in the
ASC setting associated with the lowest total medical and pharmacy health care cost (\$20,953; Supplementary Table 4, available in online article). The mean pharmacy costs were between approximately $\$ 1,000$ and $\$ 2,500$, irrespective of the venue and procedure, while the medical costs ranged from approximately $\$ 20,000$ to $\$ 90,000$.

During the first 30 days of the postsurgical period, unadjusted all-cause combined medical and pharmacy costs were $54 \%$ lower for HOPDs $(\$ 20,468)$ and $57 \%$ lower for ASCs $(\$ 19,110)$ compared with inpatient venues $(\$ 44,566$; $\mathrm{P}<0.001$; Table 2). After adjusting for demographic and baseline clinical characteristics, 30-day costs remained significantly lower for HOPDs and ASCs (14\% and 27\% lower, respectively; $\mathrm{P}<0.001$ for both) relative to the inpatient setting. Additionally, after adjusting for all covariates included in the model, total costs associated with LSF were more than twice the total medical and pharmacy costs associated with TKA (cost ratio, 2.16; $\mathrm{P}<0.001$; Table 3 ); total costs associated with RCR were approximately half the costs associated with TKA (cost ratio, 0.55; $\mathrm{P}<0.001)$. Cost ratios were significantly lower among patients aged $\geq 65$ years 


\section{TABLE 2 Two All-Cause Unadjusted Health Care Costs During the First 30 Days of the Postsurgical Period ${ }^{a}$}

\begin{tabular}{|c|c|c|c|c|c|c|c|}
\hline & $\begin{array}{c}\text { TKA } \\
(n=40,574)\end{array}$ & $\begin{array}{c}\text { PKA } \\
(n=4,355)\end{array}$ & $\begin{array}{c}\text { THA } \\
(n=25,585) \\
\end{array}$ & $\begin{array}{c}\text { TSA } \\
(n=3,133)\end{array}$ & $\begin{array}{c}\text { RCR } \\
(n=34,207) \\
\end{array}$ & $\begin{array}{c}\text { LSF } \\
(n=18,318)\end{array}$ & $\begin{array}{c}\text { All } \\
(\mathrm{N}=126,172)\end{array}$ \\
\hline \multicolumn{8}{|c|}{ Mean medical costs } \\
\hline Inpatient & 35,340 & 33,375 & 35,355 & 30,475 & 26,484 & 81,561 & 44,195 \\
\hline HOPD & 28,785 & 22,852 & 29,437 & 26,134 & 17,970 & 54,539 & 20,200 \\
\hline \multirow[t]{2}{*}{ ASC } & 29,576 & 25,638 & 31,293 & 29,947 & 14,596 & 78,350 & 18,862 \\
\hline & $P<0.001$ & $P<0.001$ & $P<0.001$ & $P=0.018$ & $P<0.001$ & $P<0.001$ & $P<0.001$ \\
\hline \multicolumn{8}{|c|}{ Mean pharmacy costs } \\
\hline Inpatient & 388 & 366 & 326 & 344 & 548 & 402 & 371 \\
\hline HOPD & 369 & 325 & 228 & 249 & 257 & 374 & 267 \\
\hline \multirow[t]{2}{*}{ ASC } & 368 & 231 & 190 & 252 & 239 & 357 & 248 \\
\hline & $P=0.810$ & $P=0.004$ & $P<0.001$ & $P=0.106$ & $P<0.001$ & $P=0.458$ & $P<0.001$ \\
\hline \multicolumn{8}{|c|}{ Mean medical and pharmacy costs combined } \\
\hline Inpatient & 35,728 & 33,741 & 35,680 & 30,819 & 27,032 & 81,963 & 44,566 \\
\hline HOPD & 29,154 & 23,177 & 29,665 & 26,383 & 18,227 & 54,913 & 20,468 \\
\hline \multirow[t]{2}{*}{ ASC } & 29,945 & 25,869 & 31,483 & 30,199 & 14,836 & 78,707 & 19,110 \\
\hline & $P<0.001$ & $P<0.001$ & $P<0.001$ & $P=0.015$ & $P<0.001$ & $P<0.001$ & $P<0.001$ \\
\hline
\end{tabular}

Note: All $P$ values are the result of analysis of variance across surgical venues and do not indicate statistical significance between any 2 venues.

${ }^{a}$ All costs are represented in U.S. dollars.

$A S C=$ ambulatory surgical center; $H O P D=$ hospital outpatient department; $L S F=$ lumbar spinal fusion; $P K A=$ partial knee arthroplasty; $R C R=$ rotator cuff repair; $T H A=$ total hip arthroplasty; TKA = total knee arthroplasty; TSA = total shoulder arthroplasty.

relative to those in patients aged $\leq 34$ years (cost ratio for ages $65-74$ years, $0.72[\mathrm{P}<0.001]$; cost ratio for age $\geq 75$ years, $0.23[\mathrm{P}<0.001])$.

Similar findings were found in analyzing adjusted cost ratios for 90-day costs, with significantly lower cost ratios for HOPDs and ASCs (relative to the inpatient setting), RCR (relative to TKA), and patients aged $\geq 65$ years (relative to patients aged $\leq 34$ years) as well as significantly higher cost ratios for LSF (relative to TKA; Supplementary Table 5, available in online article).

\section{Discussion}

In this retrospective analysis of commercial insurance claims, we identified $>126,000$ patients undergoing orthopedic procedures (TKA, PKA, THA, TSA, RCR, or LSF) in traditional inpatient venues, ASCs, or HOPDs. From 2012 to 2017, use of ASCs generally increased, whereas use of HOPDs and inpatient venues remained consistent. A multivariable logistic regression model adjusted for procedure cohort, surgery year, demographic characteristics, baseline QuanCharlson Comorbidity Index, and baseline long-term opioid use demonstrated that previous long-term opioid use in the
4 months before index surgery was associated with increased likelihood of developing persistent postsurgical opioid use, whereas undergoing procedures at either HOPDs or ASCs was associated with decreased likelihood of persistent postsurgical opioid use. It should be noted that individuals with long-term opioid use who were classified as having persistent postsurgical opioid use may indeed be considered as continuing a persistent opioid use that was initiated before surgery. Adjusted cost ratios were significantly lower at HOPDs and ASCs during the first 30 and 90 days of the postsurgical period compared with inpatient venues.

Together, these findings indicate that transitioning orthopedic procedures for appropriate patients (e.g., healthy patients or those with mild systemic disease per the American Society of Anesthesiologists [ASA] Physical Status Classification System) from inpatient to outpatient surgical sites such as HOPDs and ASCs may be associated with multiple benefits, including decreased likelihood of persistent opioid use and reduced health care costs. ${ }^{15}$

Although opioids are often prescribed after orthopedic surgery, ${ }^{16}$ their therapeutic benefit is complicated by risk for long-term use. Indeed, we found that persistent postsurgical opioid use was higher among opioid-experienced 


\section{TABLE 3 Adjusted Cost Ratios of 30-Day Post-Index Costs $(\mathrm{N}=126,128)^{\mathrm{a}}$}

\begin{tabular}{l|ll|l}
\hline \multicolumn{3}{|l|}{ Cost Ratio $(95 \% \mathrm{CI})$} & P Value \\
\hline Venue (ref: inpatient venue) & 0.86 & $(0.84-0.88)$ & $<0.001$ \\
\hline HOPD & 0.73 & $(0.71-0.74)$ & $<0.001$ \\
\hline ASC & \multicolumn{3}{|l}{}
\end{tabular}

Surgical procedure (ref: TKA)

\begin{tabular}{l|rr|r}
\hline PKA & 0.86 & $(0.84-0.88)$ & $<0.001$ \\
\hline THA & 0.97 & $(0.96-0.98)$ & $<0.001$ \\
\hline TSA & 0.92 & $(0.89-0.94)$ & $<0.001$ \\
\hline RCR & 0.55 & $(0.54-0.56)$ & $<0.001$ \\
\hline LSF & 2.16 & $(2.13-2.19)$ & $<0.001$ \\
\hline \multicolumn{4}{|l|}{} \\
\hline Surgery year (ref: 2012) & 1.03 & $(1.02-1.05)$ & $<0.001$ \\
\hline 2013 & 1.03 & $(1.02-1.05)$ & $<0.001$ \\
\hline 2014 & 1.03 & $(1.02-1.05)$ & $<0.001$ \\
\hline 2015 & 1.02 & $(1.00-1.03)$ & 0.027 \\
\hline 2016 & 1.02 & $(1.01-1.03)$ & 0.007 \\
\hline 2017 & & &
\end{tabular}

Age, years (ref: $18-34$ years)

\begin{tabular}{l|rr|r}
\hline $35-54$ & 0.98 & $(0.95-1.01)$ & 0.155 \\
\hline $55-64$ & 0.97 & $(0.94-1.00)$ & 0.063 \\
\hline $65-74$ & 0.72 & $(0.70-0.74)$ & $<0.001$ \\
\hline$\geq 75$ & 0.23 & $(0.22-0.24)$ & $<0.001^{\mathrm{b}}$ \\
\hline Sex (ref: female) & 1.03 & $(1.02-1.04)$ & $<0.001$ \\
\hline Male & \multicolumn{3}{|l}{} \\
\hline
\end{tabular}

\begin{tabular}{l|ll|l}
\hline Region (ref: South) & & \multicolumn{3}{l}{} & \\
\hline Northeast & 1.18 & $(1.16-1.20)$ & $<0.001$ \\
\hline Midwest & 0.95 & $(0.94-0.95)$ & $<0.001$ \\
\hline West & 1.07 & $(1.06-1.08)$ & $<0.001$ \\
\hline
\end{tabular}

Baseline Quan-Charlson Comorbidity Index (ref: 0)

\begin{tabular}{l|rr|r}
\hline $1-2$ & 1.01 & $(1.00-1.02)$ & 0.012 \\
\hline$\geq 3$ & 1.06 & $(1.04-1.08)$ & $<0.001$ \\
\hline $\begin{array}{l}\text { Any opioid use in the last 90 days } \\
\text { during the baseline period } \\
\text { (ref: no opioid use during this time) }\end{array}$ & 1.03 & $(1.02-1.04)$ & $<0.001$ \\
\hline
\end{tabular}

"The 44 patients with region set as "other" were excluded from the model. ${ }^{b}$ Because the sample was drawn from a commercially insured population, and costs reflect insurer- and patient-paid amounts, the lower costs for individuals aged $\geq 65$ years do not include amounts paid by Medicare.

$A S C=$ ambulatory surgical center; $H O P D=$ hospital outpatient department; $L S F=$ lumbar spinal fusion; $P K A=$ partial knee arthroplasty; $R C R=$ rotator cuff repair; ref = reference; THA = total hip arthroplasty; TKA = total knee arthroplasty; TSA = total shoulder arthroplasty. patients compared with opioid-naive patients, potentially because patients were already initiated on opioids for underlying conditions. This is in line with previous studies reporting that exposure to opioids is a risk factor for persistent postsurgical use, diversion, and overdose. ${ }^{17-19} \mathrm{We}$ also found that odds of persistent postsurgical opioid use were lower in ASCs and HOPDs than inpatient venues. This finding was consistent with the proportion of patients with persistent opioid use when stratified by baseline versus no baseline long-term use in different surgical venues. Although the direct cause of this difference cannot be assessed with a retrospective study design of available data from commercial insurance claims, possible explanations include patients reporting significantly better quality of education about postsurgical recovery and facility support for managing pain at ASCs relative to inpatient venues. ${ }^{20}$ Additional studies comparing quality of education initiatives and medical support provided at outpatient sites with that at inpatient settings would provide a better understanding of how surgical venues directly affect opioid use after surgery.

We also found that adjusted all-cause health care costs during the postsurgical period for orthopedic procedures performed at ASCs or HOPDs were significantly lower compared with inpatient venues. These cost differences are primarily explained by those accrued on the day of surgery through 30 days after surgery, given that cost ratios for 90 days were similar to the 30-day estimates. These results align with a previous study that found lower costs of combined surgical and postsurgical care up to 90 days for ASCs compared with inpatient venues for TKA (13\%) and THA (15\%). ${ }^{8}$ Interestingly, this previous study also found that surgical and postsurgical costs of TKA and THA were higher in ASCs compared with HOPDs ( 5\% for both procedures). ${ }^{8}$

Taken together, the findings from the current retrospective analysis of $>126,000$ patients support previous reports among smaller samples indicating that shifting more procedures to HOPDs or ASCs from inpatient settings can reduce medical costs. Future research using large claims databases or other sizable real-world sources can confirm whether these trends will continue.

\section{LIMITATIONS}

This study has some limitations to consider. The surgical cohorts were not assigned randomly, and choice of venue can depend on many patient, physician, and payer factors that may influence outcomes. While multiple observed confounders were controlled for in the multivariable regression analysis, results remained subject to unobserved bias. We also relied on available data from commercial insurance 
claims to assess 1 aspect of patient postsurgical recovery (postsurgical opioid use).

Claims data are intended for reimbursement; retrospective review of such data is unable to capture important patient data such as presurgery ASA Physical Status Classification or extent of postsurgical pain, which may in part explain opioid use after surgery. Understanding patient ASA status would be important for identifying patients who are surgical candidates appropriate for migration to ASCs. Race and other socioeconomic information, such as income, were also not available in the claims data.

Further, this study did not capture the history of medical conditions that can potentially influence opioid utilization in the follow-up period, beyond the Quan-Charlson Comorbidity Index. However, our findings contribute a valuable understanding of factors other than patient characteristics (i.e., surgical venue and type of procedure) in relation to postsurgical opioid use. While opioid use was assessed using prescription fills in this study, comparing opioid dosage across the groups in future studies may provide more information about opioid use patterns following these procedures across groups.

Furthermore, this study specifically used commercial health claims data, limiting the extent to which findings can be applied to populations that are publicly insured by options such as Medicaid or including patients without insurance. Because the sample was drawn from a commercially insured population, and costs reflected amounts paid by the insurer and patient, the costs associated with Medicare patients aged $\geq 65$ years were not included in the current analysis.

Finally, most of this study period took place before the dissemination of the Centers for Disease Control and
Prevention opioid prescribing guidelines. ${ }^{21}$ Because efforts to address the opioid crisis are underway, the prevalence and sequelae of opioid addiction may have changed prescribing patterns since the period of data collection for this study. However, opioids continue to be prescribed for postsurgical pain, and understanding how aspects of surgical care can mitigate risk for long-term use remains valuable.

\section{Conclusions}

Our retrospective analysis of commercial claims data indicates that patients undergoing orthopedic surgical procedures at HOPDs and ASCs were less likely to develop persistent postsurgical opioid use compared with patients undergoing these procedures at inpatient venues. Additionally, these procedures were associated with lower health care costs, supporting the transition of performing orthopedic procedures from inpatient venues to ASCs and HOPDs for both clinical and economic benefit for eligible patients.

\section{DISCLOSURES}

This study and editorial support for the preparation of this manuscript was funded by Pacira BioSciences, which contracted with Optum to conduct the study. Cisternas, Korrer, and Wilson are employees of Optum. Waterman was employed with Pacira BioSciences at the time of the study.

Portions of this work were presented at AMCP Nexus 2019; October 29-November 1, 2019; National Harbor, MD.

\section{ACKNOWLEDGMENTS}

Editorial assistance was provided by Paul Cavanaugh, PhD, and Mary Helen Tran, former employees of Pacira BioSciences, and Susan F. Peckous and Rachel Halpern, employees of Optum HEOR. Editorial assistance was also provided under the direction of the authors by Caroline Jennermann of Optum HEOR and Aarthi Gobinath, PhD, and David Boffa, ELS, of MedThink SciCom.

\section{REFERENCES}

1. Ambulatory Surgery Center Association. A positive trend in healthcare. Accessed January 12, 2021. https://higherlogicdownload.s3.amazonaws.com/ ASCACONNECT/fd1693e2-e4a8-43d3816d-17ecfc7d55c1/UploadedImages/ About\%20Us/ASCs\%20-\%20A\%20 Positive\%20Trend\%20in\%20Health\%20 Care.pdf

2. Dykens EM, Maxwell MA, Pantino E, Kossler R, Roof E. Assessment of hyperphagia in Prader-Willi syndrome. Obesity (Silver Spring). 2007;15:1816-26.

3. Beaussier M, Sciard D, Sautet A. New modalities of pain treatment after outpatient orthopaedic surgery. Orthop Traumatol Surg Res. 2016;102

(1 Suppl):S121-24.

4. Memtsoudis SG, Kuo C, Ma Y, Edwards A, Mazumdar M, Liguori G. Changes in anesthesia-related factors in ambulatory knee and shoulder surgery: United States 1996-2006. Reg Anesth Pain Med. 2011;36:327-31.

5. Olderog A. Trends in total joint replacement. ASC Focus. May 2019. Accessed January 12, 2021. https:// www.ascfocus.org/ascfocus/content/ articles-content/articles/2019/may/ trends-in-total-joint-replacement

6. Aynardi M, Post Z, Ong A, Orozco F, Sukin DC. Outpatient surgery as a means of cost reduction in total hip arthroplasty: a case-control study. HSS J. 2014:10:252-55.

7. Richter DL, Diduch DR. Cost comparison of outpatient versus inpatient unicompartmental knee arthroplasty. Orthop J Sports Med. 2017;5:2325967117694352. 
8. Carey K, Morgan JR, Lin MY, Kain MS, Creevy WR. Patient outcomes following total joint replacement surgery: a comparison of hospitals and ambulatory surgery centers. J Arthroplasty. 2020;35:7-11.

9. Crawford DC, Li CS, Sprague S, Bhandari M. Clinical and cost implications of inpatient versus outpatient orthopedic surgeries: a systematic review of the published literature. Orthop Rev (Pavia). 2015;7:6177.

10. Martin-Ferrero MA, Faour-Martin O, Simon-Perez C, Perez-Herrero M, de Pedro-Moro JA. Ambulatory surgery in orthopedics: experience of over 10,000 patients. J Orthop Sci. 2014;19:332-38.

11. Society of Hospital Medicine. Multimodal pain strategies guide for postoperative pain management. 2017. Accessed January 12, 2021. https:// www.hospitalmedicine.org/globalassets/clinical-topics/clinical-pdf/ ctr-17-0004-multi-model-pain-projectpdf-version-m1.pdf
12. Gerbershagen HJ, Aduckathil S, van Wijck AJ, Peelen LM, Kalkman CJ, Meissner W. Pain intensity on the first day after surgery: a prospective cohort study comparing 179 surgical procedures. Anesthesiology. 2013;118:934-44.

13. Quan H, Li B, Couris CM, et al. Updating and validating the Charlson Comorbidity Index and Score for risk adjustment in hospital discharge abstracts using data from 6 countries. Am J Epidemiol. 2011;173:676-82.

14. U.S. Bureau of Labor Statistics. Measuring price change in the $\mathrm{CPI}$ : medical care. November 2020. Accessed January 12, 2021. https://www.bls.gov/ cpi/factsheets/medical-care.htm

15. College of Physicians and Surgeons of British Columbia. ASA physical status classification guideline. December 30, 2017. Accessed January 12, 2021. https:// www.cpsbc.ca/files/pdf/NHMSFAP-ASAPhysical-Status-Classification.pdf
16. Lipof JS, Thirukumaran CP,

Greenstein AS, Zmich Z, Lander A, Ricciardi BF. Postdischarge opiateprescribing habits for primary THA and TKA: a survey of American Association of Hip and Knee Surgeons members. Orthopedics. 2019;42:361-67.

17. Kim SC, Choudhry N, Franklin JM, et al. Patterns and predictors of persistent opioid use following hip or knee arthroplasty. Osteoarthritis Cartilage. 2017;25:1399-406

18. Vu JV, Lin LA. Opioid overdose-the surgeon's role. Ann Surg. 2018;268:32-34.

19. Wojahn RD, Bogunovic L, Brophy $\mathrm{RH}$, et al. Opioid consumption after knee arthroscopy. J Bone Joint Surg Am. 2018;100:1629-36.

20. Kelly MP, Calkins TE, Culvern C, Kogan M, Della Valle CJ. Inpatient versus outpatient hip and knee arthroplasty: which has higher patient satisfaction? J Arthroplasty. 2018;33:3402-06.

21. Dowell D, Haegerich TM, Chou R. CDC guideline for prescribing opioids for chronic pain - United States, 2016. MMWR Recomm Rep. 2016;65:1-49. 